Gauss Clock, Gauss Line and Magnet Integration

$$
A G S
$$

Booster Technical Note

$$
\text { No. } 213
$$

\author{
B.B. Culwick \\ July 301992
}

ALTERNATING GRADIENT SYNCHROTRON DEPARTMENT

BROOKHAVEN NATIONAL LABORATORY

UPTON, N.Y. 11973 
Booster Technical Note No. 213

\section{Gauss Clock, Gauss Line and Magnet Integration}

\section{Introduction}

This note is an attempt to summarize the state of the Booster Gauss Clock, Gauss Line and Main Magnet Power Supply regulation and to list the remaining items to complete the system.

\section{Gauss Clock}

The Booster Gauss Clock [1] has now been running for some months. It has provided useful signals to many pieces of equipment and has been crucial in defining injection, extraction and $R F$ frequency. Some questions remain regarding:

\subsection{Calibration}

There is some question regarding the calibration of frequency versus $B$ dot for the clock. This will probably be addressed by the RF group which is most sensitive to the effects.

The drift of the Gauss Clock ( finite output frequency at zero B dot ) is small but significant corresponding to about 1 gauss per second.

The initial values of the gauss registers in various equipment are set from the Gauss Line Generator. However, the GLG itself needs a calibration value (See below).

\section{Gauss Line Generator}

\subsection{Operation}

The Gauss Line Generator [2] has also operated satisfactorily for some months.

\subsection{BGT.CALIBRATE}

The absolute value of the Gauss Register in the Gauss Line Generator is established by jamming a value into the register at a time determined by a Super Cycle event. The value to be jammed is entered manually at present by a procedure referring to a hall probe measurement in the refence magnet. This procedure should be made automatic and a number of proposals to achieve this have been discussed [3]. A resolution of the options should be made and significant effort may be required to implement the selected solution.

\subsection{BGT.SFIELD.BUFFER}

The initialization of the gauss event table is presently achieved by the field value passing through an event which is the lowest value in the table. This procedure imposes 
some constraints on the operating cycle and requires judicious selection of event positioning. I believe that a procedure which allows initialization of the event table at a suitable time (BT0?) can be derived without constraints on the operation except to assume a modest rate of the Gauss Clock at initialization time. The procedure would require reading the value of the gauss register (not possible at present) and searching the event table to find an appropriate setting of the table pointer. The gauss register would then be read again to verify that the pointer value was still valid. If it were not, the procedure would be repeated until a success was achieved. Since the gauss clock frequency at a suitable time is $<1 \mathrm{KHz}$ and gauss events are typically 10's of gauss apart, many $\mathrm{mS}$ are available for the procedure which will usually succeed the first time.

\section{Booster Main Magnet}

The Apollo model of the Booster Main Magnet now uses cycle dependent parameters and is able to produce cycles predictable and consistent to a few tenths of $1 \%$. Some innovative work on regulation of the dwell with an intelligent sampled servo has produced average dwell values accurate and stable to a few parts in $10^{5}$ [4]. If this technique is extended to regulation over the whole cycle, it is possible that the magnetic field will be more stable than the gauss clock. In this case, real-time triggers would be more precise than gauss triggers and the virtues of the gauss clock technique are negated. However, we are sufficiently committed to the gauss clock approach that it is impractical to change now. Sufficient support must be provided to make the gauss clock accuracy adequate for our needs.

\section{Synthetic Gauss Clock}

A proposal to provide a backup for the Booster Gauss Clock, now referred to as the Synthetic Gauss Clock was made in March 1991 [5].

The basic hardware to implement the proposal is available but no code has been written yet.

\subsection{BTN 210 Proposal}

This proposal implements the synthetic clock using modified vector generator boards to convert the B dot to a frequency simulating the real Gauss Clock (See reference 5 for details). The synthetic clock is PPM supporting the standard four users. A subtlety in operation would be to permit one or more users to be synthetic whilst other users were real. A complexity of the approach implementing this feature is that the real Gauss Clock would be inhibited during the Synthetic Gauss Clock operation. To correct for lost counts during this time, the calibration procedure described above would have to be exercised on transfer from Synthetic to Real Gauss Clock.

Some peripheral details of the Synthetic Gauss Clock remain to be resolved, for example the required magnetic field range is 13 Kgauss which is represented by 130000 counts in the Booster and perhaps 260000 counts in the AGS. The vector generator supports only a 16-bit counter so some ingenuity is called for if the function is required in addition to its derivative. The function would be useful in a comfort display and for 
diagnostic monitoring.

\section{A Synthetic Gauss Line}

All users except the low-level Radio Frequency would be served by a Synthetic Gauss Line since they make no direct use of the Gauss Clock. One advantage of a Synthetic Gauss Line is that it can be implemented as PPM without compromising the calibration of the Real Gauss Clock. An interesting facet of a Synthetic Gauss Line is that the data required for a simulation of the present supercycle is available from the Gauss Line Monitor in the form of the time-stamped Gauss Events. This data would allow simulation of operational Gauss Line cycles in a straightforward manner. Test and modified cycles would require some system support to create the necessary tables.

These virtues must be weighed against the limitations that the SGL would provide no support for low-level RF frequency synthesis and would not test the early parts of Gauss Clock processing. The latter aspect is a mixed blessing since the SGL would not be dependent on operation of those system components, allowing development and testing of them.

The hardware required by a SGL would be essentially a duplicate of the existing Gauss Line Generator board driven by a real-time clock.

[1] J.Geller, The Booster Gauss Clock, Booster Technical Note \#175

[2] B. Culwick, Memorandum: Booster Gauss Line Generator 12/27/89

[3] B. Culwick, Absolute Calibration of the Booster Gauss Clock, Booster Technical Note \#186

[4] J. Geller, Private Communication

[5] B. Culwick, Backup for the Booster Gauss Clock, Booster technical Note \#210 